Ciencia y Educación, Vol. 4, No. 3, septiembre-diciembre, 2020

ISSN (impreso): 2613-8794•ISSN (en línea): 2613-8808

DOI: https://doi.org/10.22206/cyed.2020.v4i3.pp95-113

\title{
La tesis y la formación en investigación: significados construidos por estudiantes de pedagogía
}

\author{
Thesis and research training: meanings constructed \\ by pedagogy students
}

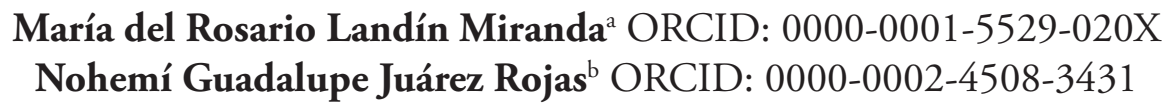

Recibido: 29/02/2020 • Aprobado: 18/05/2020

Cómo citar: Landín Miranda, M. del R., \& Juárez Rojas, N. G. (2020). La tesis y la formación en investigación: significados construidos por estudiantes de pedagogía. Ciencia y Educación, 4(3), 95-113. Doi: https://doi.org/10.22206/cyed.2020.v4i3.pp95-113

\section{Resumen}

La formación en Investigación Educativa es un aspecto fundamental en la Licenciatura en Pedagogía de la Universidad Veracruzana, México, en congruencia con la política educativa a nivel nacional. En ese contexto, se desarrolló un estudio cualitativo a través de entrevistas a profundidad a ocho estudiantes universitarios. La metodología de las comparaciones constantes y el programa Atlas Ti, permitieron integrar categorías con el fin de explorar aquellos significados que han construido sobre la formación en investigación educativa y la tesis. El estudio permitió conocer cómo el contenido de los significados es el producto de la interacción que los estudiantes establecen con sus iguales y los profesores, con el currículum, el contexto escolar y social. En conclusión, la formación en investigación educativa requiere formar parte del desarrollo curricular; ser vista como experiencia, donde profesor y estudiante se impliquen en la búsqueda incesante de la pregunta, en la aventura por conocer.

Palabras clave: investigación educativa; pedagogía; estudiantes; currículum; experiencia.

\begin{abstract}
In accordance with educational policy at the national level, training in Educational Research is a fundamental aspect in the Bachelor of Pedagogy at the Universidad Veracruzana, Mexico. In this context, we developed a qualitative study, through in-depth interviews with eight university students. The methodology of constant comparisons and the Atlas Ti allowed categories to be integrated in order to explore those meanings that they have built on educational research training and the thesis. The study allowed to know how the content of meanings is the product of the interaction that students establish with their peers and teachers, with the curriculum, the school and social context. In conclusion, training in educational research requires being part of the curriculum development; it needs to be seen as an experience, where the teacher and student are involved in the incessant search for the question, in the adventure to know.
\end{abstract}

Keywords: educational research; pedagogy; students; curriculum; experience.

\footnotetext{
a Universidad Veracruzana, México

Correo-e: rlandin@uv.mx

b Universidad Veracruzana, México.

Correo-e: zS16005395@estudiantes.uv.mx
} 


\section{Introducción}

La formación en investigación educativa sigue siendo un tema pendiente no solo desde la política educativa en México, sino por las diversas Instituciones de Educación Superior (IES). El fortalecimiento del quehacer científico en torno a la educación superior es prioritario para atender los problemas educativos e innovar en las aulas; para el financiamiento y el desarrollo curricular que permita dar continuidad a la formación en investigación educativa desde la licenciatura hasta el posgrado.

Desde este contexto, en la Licenciatura en Pedagogía de la Universidad Veracruzana, México, se considera la investigación educativa parte fundamental en la formación de los estudiantes. El plan de estudios (2000) contempla una serie de cursos sobre metodología de la investigación cuantitativa y cualitativa para que un estudiante pueda desarrollar un trabajo de investigación que le permita la integración de un trabajo recepcional y obtenga el grado. La elaboración del trabajo recepcional forma parte de la malla curricular con valor crediticio, lo que favorece la titulación del estudiante y la eficiencia terminal. Sin embargo, se ha observado que los estudiantes no culminan la cadena del Área de Investigación Educativa, la cual termina con el curso de Experiencia Recepcional y la presentación formal de su tesis, inclinándose por otras opciones de titulación que se consideran en dicha universidad, específicamente en el Estatuto de los alumnos 2008, donde se establece el derecho que otorga al universitario para elegir su proceso de titulación:

Artículo 78. Los alumnos que cursen planes de estudio flexibles de nivel técnico y de estudios profesionales podrán acreditar la experiencia recepcional a través de las siguientes opciones:

I. Por trabajo escrito presentado en formato electrónico bajo la modalidad de tesis, tesina, monografía, reporte o memoria y las demás que apruebe la Junta Académica de cada programa educativo;
II. Por trabajo práctico, que puede ser de tipo científico, educativo, artístico o técnico;

III. Por promedio, cuando hayan acreditado todas las experiencias educativas del plan de estudios con promedio ponderado mínimo de 9.00 en ordinario en primera inscripción, en los casos que así lo apruebe la Junta Académica;

IV. Por examen general de conocimientos. (Universidad Veracruzana, 2008, pp. 28-29)

Ante estas alternativas de titulación, se ha observado que la mayoría de los estudiantes eligen la modalidad por promedio. Esta modalidad significa que el estudiante obtuvo una calificación excelente durante toda la carrera, sin cursos reprobatorios, lo que le permite obtener su titulación sin necesidad de realizar la tesis. Aunque tal situación favorece al estudiante, contraviene con los propósitos del área de investigación educativa que integra el plan de estudios de Pedagogía, pues el estudiante no vive la experiencia de hacer investigación; una experiencia para desarrollar y poner en práctica sus habilidades indagativas que le permitan fortalecer su formación académica y profesional. Investigar forma parte del acto de estudiar, implica, en palabras de Freire (2011): "un trabajo difícil. Exige de quien lo hace una actitud crítica, sistemática. Exige una disciplina intelectual que no se adquiere sino practicándola” (p.48).

En los espacios académicos de la Facultad de Pedagogía se ha escuchado, entre la mayoría de los estudiantes, que la tesis es un requisito institucional, un escrito que debe de cumplir con determinado número de cuartillas. Sin embargo, la tesis implica un proceso indagativo, argumentativo, que favorece el desarrollo de habilidades analíticas y reflexivas, pues "en cuanto más ponemos en práctica de manera metódica nuestra capacidad de indagar, de comparar, de dudar, de verificar, tanto más eficazmente curiosos nos podemos volver" (Freire, 2018, p. 61). Sin lugar a duda, el proceso indagativo tiene como base fundamental la curiosidad. Al respecto, Freire (2018) hace 
una interesante aportación: "el ciclo gnoseológico parte de la curiosidad que, al volverse cada vez más metódicamente rigurosa, transita de la ingenuidad hacia lo que él llama "curiosidad epistemológica" (p. 31).

La tesis es una oportunidad para fortalecer la curiosidad epistemológica, pues "la disposición a conocer, la apertura a explorar, la curiosidad por las cosas es lo que alimenta a la investigación" (Contreras y Pérez, 2013, p. 63). La tesis y la investigación desde la experiencia de Caramés (2013) "son nada más, aunque también nada menos, que espacios posibles para aprender" (p. 202).

El estudiante debe aprovechar su estancia académica y las experiencias que desde ella se generan en la etapa universitaria, pues quizá sea la primera oportunidad que tenga para vivir experiencias académicas como publicar, participar en eventos académicos, hacer estancias de investigación, favoreciendo la madurez y el buen ejercicio profesional.

Esta situación y el interés por fortalecer la formación en investigación educativa, ha llevado a realizar el presente estudio, el cual fue focalizado a estudiantes de $7^{\circ}$ semestre del plan de estudios 2000 generación 2016-2019 de la Facultad de Pedagogía, de la Universidad Veracruzana Región Poza Rica, México.

\section{Marco teórico}

\section{La teoría del interaccionismo simbólico de Herbert Blumer}

El posicionamiento teórico para el desarrollo del presente estudio está basado en la teoría del Interaccionismo Simbólico de Herbert Blúmer, dado que nos permite comprender cómo los significados construidos por las personas direccionan su actuar, donde su manera de interpretar juega un papel fundamental en dicha construcción. Al respecto Taylor y Bogdan (2000) comentan que, "el proceso de interpretación es un proceso dinámico. La manera en que una persona interprete algo dependerá de los significados de que se disponga y de cómo se aprecie una situación” (p. 25).
Estos mismos autores afirman que, "desde una perspectiva interaccionista simbólica, todas las organizaciones, culturas y grupos están constituidos por actores envueltos en un proceso constante de interpretación del mundo que los rodea” (p. 25).

El interaccionismo simbólico se basa en tres premisas:

- La primera es que el ser humano orienta sus actos hacia las cosas en función de lo que estas significan para él.

- La segunda premisa es que el significado de estas cosas se deriva de, o surge como consecuencia de la interacción social que cada cual mantiene con el prójimo.

- La tercera es que los significados se manipulan y modifican mediante un proceso interpretativo desarrollado por la persona al enfrentarse con las cosas que va hallando a su paso (Blumer, 1982, p. 2)

Estas premisas se mueven desde lo que Blúmer ha llamado imágenes radicales, las cuales son: "sociedad o grupos humanos, interacción social, objetos, el ser humano como agente, los actos humanos y la interconexión de las líneas de acción” (p. 5). En conjunto, estas imágenes radicales son la base fundamental del interaccionismo simbólico que permiten el análisis del comportamiento y la sociedad.

El interaccionismo simbólico da los elementos para comprender cómo los estudiantes, en interacción con sus iguales, el contexto escolar, familiar y social, construyen y reconstruyen sus significados, basándose en un proceso de interpretación donde se juegan intereses personales y profesionales, que llevan a orientar su propia actuación y toma de decisiones.

\section{El docente - investigador}

La propuesta de Lawrence Stenhouse, el profesor-investigador, es congruente con la mirada del presente estudio. Los orígenes de esta figura se 
remontan a un proyecto impulsado por dicho autor: Humanities Curriculum Project (HPC), en el cual se considera a un profesor impulsor del desarrollo no solo del currículum, sino del desarrollo profesional, donde la investigación es la base fundamental para lograrlo (Stenhouse, 2004).

Estas ideas, permiten ver claramente cómo la investigación forma parte del quehacer docente, de la formación y del desarrollo curricular. Un profesor, de acuerdo con Stenhouse (2004) "se halla rodeado por abundantes oportunidades de investigar" (p.38). Por otro lado, Cochran-Smith y Lytle (2002) afirman que los profesores:

poseen un conocimiento amplio de la vida y la cultura de la comunidad social, escolar y del aula a la que pertenecen y además experimentan los acontecimientos de la vida del aula en relación a sus propios roles y responsabilidades (pp. 41-42).

Esta situación en la que viven los profesores les permite fortalecer sus propias herramientas y generar saberes pertinentes sobre cuestiones educativas.

En este sentido, los profesores que hacen investigación, con su ejemplo y práctica, pueden contribuir con la formación de los estudiantes, compartir experiencias de lo que han investigado, investigan e investigarán, tal proceder se convierte en una fuente importante de aprendizajes desde la práctica, abriendo espacios favorables para la investigación que impulsa el desarrollo curricular y favoreciendo de manera recursiva todo lo anterior.

La propuesta de Lawrence Stenhouse, la investigación es base de la enseñanza, es congruente con uno de los principios pedagógicos que observamos desde el presente estudio y que argumenta acertadamente Paulo Freire: enseñar exige investigación, "no hay enseñanza sin investigación ni investigación sin enseñanza”. Y precisa que, "La indagación, la búsqueda, la investigación, forman parte de la naturaleza de la práctica docente" (Freire, 2018, p. 30).

\section{La investigación vista como experiencia}

Otro aspecto que apoya el posicionamiento teórico del estudio, es considerar la investigación como experiencia, pero no como simple experiencia acumulativa de actividades o conocimientos. El sentido de la experiencia que aquí se aborda es, en palabras de Contreras y Pérez (2013):

lo vivido que deja huella, que hace un efecto en nosotros. Más que con la voluntad, tiene que ver con la subjetividad y con la consciencia: la relación que se da entre aquello en lo que uno (o una) está inmerso y la forma en que se ve implicado, concernido, afectado, llamado, reclamado, sorprendido, desconcertado, (...) (p. 34).

Este sentido de experiencia lleva a mirar de forma diferente la investigación educativa. No vista como un conjunto de procedimientos, de técnicas para explicar la realidad, más bien esa experiencia es la búsqueda incesante de la pregunta, de dejarse atrapar por el misterio, de aventurarse a lo inexplicable, a lo imprevisible; investigar en educación nos lleva a adentrarnos a vivir la experiencia desde las aulas, desde la relación con el otro; es saber mirar desde ella develando la trama de lo educativo. Entender a la investigación como experiencia es:

vislumbrar y alumbrar nuevas prácticas investigadoras, (...) supone emprender la aventura del conocimiento con la esperanza de vivir una experiencia de saber, la experiencia de aprender. Es adentrarse en una realidad desde la propia subjetividad, tratar de enfrentarse al misterio de lo que no se conoce, lo que no se comprende, e intentar ver cuáles son las preguntas que en tal situación se nos abren (Contreras y Pérez, 2013, p. 67).

Tal forma de mirar a la investigación educativa exige el compromiso de propiciar la experiencia en los espacios áulicos, donde profesor y estudiante se impliquen como aprendices que arriesgan y se equivocan; que comparten preguntas y desasosiegos 
provocados por la incertidumbre que genera indagar a través de las diversas realidades educativas.

\section{Metodología}

El objetivo del estudio fue conocer los significados que el estudiante de Pedagogía ha construido en torno a la investigación educativa y la tesis, teniendo la siguiente pregunta de investigación: ¿qué significados ha construido el estudiante de pedagogía en torno a la tesis y la investigación educativa?

La metodología utilizada para el desarrollo de esta investigación tuvo como base el enfoque cualitativo, el cual permitió considerar que son los sujetos quienes, en relación con su mundo, pueden vivir una diversidad de experiencias en determinado tiempo y lugar, construyendo sentidos y significados, pues la investigación cualitativa según Flick (2012) "se orienta a analizar casos concretos en su particularidad temporal y local, y a partir de las expresiones y actividades de las personas en sus contextos locales" (p. 27).

La tarea al desarrollar una metodología con enfoque cualitativo ha sido el cuidado de una mirada in situ, puesto que únicamente desde "dentro" es como pueden captarse y comprenderse aquellos significados profundos que estructuran la realidad en la que se encuentra inmerso un sujeto. En este sentido, Taylor y Bogdan (2000) mencionan que la metodología cualitativa es un modo de encarar el mundo empírico, lo que lleva a considerar, entre otros aspectos, que es inductiva dado que:

los investigadores desarrollan conceptos, intelecciones y comprensiones partiendo de pautas de los datos, y no recogiendo datos para evaluar modelos, hipótesis o teorías preconcebidos, [por otro lado], los investigadores cualitativos tratan de comprender a las personas dentro del marco de referencia de ellas mismas (p. 20).

Las investigadoras cualitativas que realizaron la presente investigación, han tenido la oportunidad de estar dentro del contexto de estudio desplegando "una amplia gama de métodos interpretativos interconectados, buscando siempre nuevos modos de hacer entendibles los mundos de experiencia estudiados" (Denzin y Lincoln, 2011, p. 83).

Aprovechando nuestros roles como profesora investigadora y estudiante en proceso de formación en investigación educativa, congruentes con las propuestas de Stenhouse (2003) la investigación realizada por el profesor, y de Cochram-Smith y Lytle (2002) la investigación desarrollada por enseñantes, se pudo establecer una metodología que abriera la posibilidad para la construcción del saber pedagógico, conjugando experiencias y miradas para entablar una doble reflexividad durante el estudio.

Por otro lado, como actoras e investigadoras del currículum, se pudo comprender cómo un currículum, cuando se pone en interacción constante con los estudiantes, les lleva a vivir experiencias, que son la base para la construcción de significados en torno a la formación de investigadores educativos.

\section{Sujetos de estudio}

Para el presente estudio se seleccionaron, bajo criterios intencionados, a estudiantes de la Licenciatura en Pedagogía de la Universidad Veracruzana Región Poza Rica-Tuxpan, tomando en cuenta las siguientes características:

- Formar parte de la generación 2016-2019 y que estuvieran cursando el $7^{\circ}$ semestre de la licenciatura.

- Haber tomado el curso: proyecto de investigación educativa, donde el estudiante decide si se titula por promedio o por tesis.

- Estudiantes que, de acuerdo con el Estatuto de los Alumnos 2008, eligieron la modalidad de titulación por promedio y estudiantes que llevaron el curso de Experiencia Recepcional para desarrollar la tesis de licenciatura.

- Tener la disponibilidad para participar en el estudio. 
De la generación 2016-2019 integrada por 17 estudiantes, solo participaron en el estudio 8:5 estudiantes ( 3 mujeres y 2 hombres) que realizaron el trabajo recepcional (tesis), y 3 estudiantes (2 mujeres y 1 hombre) que decidieron la titulación por promedio. Sus edades oscilaron entre 21 y 22 años.

\section{Entrevista cualitativa}

Una de las técnicas utilizadas para obtener información, fue la entrevista cualitativa que, de acuerdo con Kvale (2011) "es un camino clave para explorar la forma en que los sujetos experimentan y entienden su mundo. Proporciona un acceso único al mundo vivido de los sujetos, que describen en sus propias palabras sus actividades experiencias y opiniones" (p. 32).

Continuando con el mismo autor, la entrevista "trata de entender el significado de los temas centrales en el mundo vivido de los sujetos" (Kvale, 2011, p. 34), siendo una oportunidad para observar de manera directa la expresión corporal y emocional del estudiante. Por otro lado, Taylor y Bodgan (2000) mencionan que:

Por entrevistas cualitativas en profundidad entendemos reiterados encuentros cara a cara entre el investigador y los informantes, encuentros éstos [sic] dirigidos hacia la comprensión de las perspectivas que tienen los informantes respecto de sus vidas, experiencias o situaciones, tal como las expresan con sus propias palabras. Las entrevistas en profundidad siguen el modelo de una conversación entre iguales, y no de intercambio formal de preguntas y respuestas (p. 101).

Para el desarrollo de la entrevista se consideraron los siguientes aspectos:

- La integración de las preguntas. Se tuvo presente el planteamiento del problema, las observaciones in situ, la pregunta de investigación y el propósito del estudio. Preguntar es un arte, por ello, se tomó en cuenta la clasificación de preguntas recomendadas por Steinar Kvale (2011) para el desarrollo de una entrevista cualitativa. En el presente estudio se consideraron las preguntas introductorias, de profundización, directas y de especificación.

- La objetividad del guion. Sabemos que el término objetividad ha sido fuertemente golpeado por el enfoque positivista, sin embargo, actualmente el avance de los estudios cualitativos supera tal situación. La objetividad aplicada en los estudios cualitativos de acuerdo con Eisner (1998), permite cuidar la cuestión ontológica del estudio y su procedimiento.

La objetividad para construir el guion se basó en los criterios establecidos por Kvale, ausencia de sesgo y como acuerdo intersubjetivo (Kvale, 2011; Aguirre, 2020). En este sentido, el guion de la entrevista se construyó en colaboración con dos profesoras de la Facultad de Pedagogía y dos profesoras externas. Además, se consideró su pilotaje, aplicándolo a dos estudiantes, para observar su consistencia y objetividad en el tipo de preguntas.

Respecto al tiempo en el desarrollo de la entrevista, se destaca que el ritmo de la entrevista fue flexible, así como el manejo del tiempo, con el propósito de ir desarrollando las preguntas para cuidar su sentido reflexivo, tratando de generar un ambiente de confianza y respeto ante los diversos puntos de vista que expresaban los estudiantes.

Las entrevistas se llevaron a cabo de manera individual, acordando con cada estudiante horario, espacio y el permiso para grabarlas en audio; tuvieron una duración entre una hora y hora y media, lo que suele complicar su registro de manera manual. La grabadora de audio permitió el rescate fiel del discurso facilitando su transcripción.

Otra de las técnicas desarrolladas fue la observación participante. Basada en notas de campo, las investigadoras llevaron un registro de situaciones al interior de la Facultad de Pedagogía, observando el comportamiento de los estudiantes, sus relaciones entre iguales, así como la interacción con profesores y administrativos. En este proceso de observación, también fue importante considerar y analizar 
los documentos institucionales, los cuales orientan el comportamiento en los contextos escolares.

Las técnicas utilizadas fueron congruentes con el enfoque de investigación, pues desde el punto de vista epistemológico, "el conocimiento es el producto de la actividad humana y, por lo tanto, no se descubre, se produce por la complejidad existente de su propia realidad intencional e interactiva en un espacio y tiempo determinadas" (Trujillo, Naranjo, Rolando y Merlo, 2019, p. 76).

\section{Análisis de la información}

Respetando el anonimato de los sujetos de estudio entrevistados y para poder identificar las respuestas de cada uno de ellos, con la información recabada fue necesario utilizar la siguiente codificación:

- E: Entrevistado

- M: Masculino

- F: Femenino

- P: Promedio
- T: Tesis

- 1, 2, 3...(número): Turno en el que fue entrevistado (a)

La información se sistematizó y analizó a través de la Metodología de la Comparación Constante (MCC) de Glaser y Strauss (1967). La MCC es:

un procedimiento analítico y sistemático general para manipular los datos y construcciones lógicas derivadas de los datos, a lo largo del proceso de investigación. Es una estrategia que combina la generación inductiva de categorías con una comparación simultánea de todos los incidentes sociales observados (Martínez, 2007, p. 74).

Esta metodología, permitió el manejo de información cuidando su análisis inductivo en relación con la pregunta de investigación. El procedimiento seguido fue: identificación de unidades de significado, el establecimiento de relaciones para la construcción de categorías atendiendo sus implicaciones y exclusiones. En total se construyeron las 15 categorías tal como se observa en el cuadro 1.

Cuadro 1. Reporte general de categorías

\begin{tabular}{|c|c|c|c|c|c|c|c|c|c|c|}
\hline \multicolumn{11}{|c|}{ Reporte general de categorías } \\
\hline \multirow{2}{*}{\multicolumn{2}{|c|}{ Categorías E1FT }} & \multicolumn{9}{|c|}{ Frecuencia } \\
\hline & & \multirow{2}{*}{$\begin{array}{c}\text { E1FT } \\
2 \\
\end{array}$} & \multirow{2}{*}{$\begin{array}{c}\text { E2MT } \\
5\end{array}$} & \multirow{2}{*}{\begin{tabular}{|c|} 
E3MT \\
2 \\
\end{tabular}} & \multirow{2}{*}{$\begin{array}{c}\text { E4FT } \\
1 \\
\end{array}$} & \multirow{2}{*}{$\begin{array}{c}\text { E5FP } \\
3 \\
\end{array}$} & \multirow{2}{*}{$\begin{array}{c}\text { E6FT } \\
2 \\
\end{array}$} & \multirow{2}{*}{$\begin{array}{c}\text { E7MP } \\
2 \\
\end{array}$} & \multirow{2}{*}{$\begin{array}{c}\text { E8FP } \\
1 \\
\end{array}$} & \multirow{2}{*}{$\begin{array}{c}\text { Total } \\
18 \\
\end{array}$} \\
\hline 1 & Actitud para la investigación & & & & & & & & & \\
\hline 2 & Autonomía para la investigación & 1 & 3 & 1 & 3 & 4 & 1 & 0 & 0 & 13 \\
\hline 3 & Consideración para la investigación & 6 & 5 & 3 & 0 & 1 & 0 & 2 & 2 & 19 \\
\hline 4 & Desarrollo de habilidades & 2 & 0 & 4 & 1 & 0 & 1 & 1 & 1 & 10 \\
\hline 5 & Dificultad para hacer investigación & 2 & 7 & 6 & 1 & 9 & 4 & 1 & 5 & 35 \\
\hline 6 & Director de tesis & 1 & 2 & 1 & 1 & 0 & 1 & 0 & 0 & 6 \\
\hline 7 & Estrategias de enseñanza & 2 & 2 & 3 & 2 & 0 & 1 & 0 & 1 & 11 \\
\hline 8 & Formación desde el curriculum & 3 & 4 & 4 & 0 & 1 & 0 & 2 & 2 & 16 \\
\hline 9 & Formación terminal & 5 & 4 & 6 & 2 & 2 & 2 & 2 & 2 & 25 \\
\hline 10 & La investigación base en la formación & 6 & 7 & 6 & 5 & 4 & 4 & 4 & 3 & 39 \\
\hline 11 & La tesis & 2 & 7 & 7 & 8 & 0 & 8 & 0 & 0 & 32 \\
\hline 12 & Práctica de la investigación & 7 & 8 & 5 & 5 & 5 & 5 & 3 & 4 & 42 \\
\hline 13 & Profesor - investigador & 4 & 3 & 3 & 4 & 2 & 3 & 2 & 1 & 22 \\
\hline 14 & Significado de la investigación & 3 & 3 & 5 & 3 & 8 & 7 & 4 & 4 & 37 \\
\hline 15 & Tema de investigación & 3 & 9 & 3 & 2 & 0 & 0 & 0 & 1 & 18 \\
\hline \multicolumn{2}{|c|}{ Total } & 49 & 69 & 59 & 38 & 39 & 39 & 23 & 27 & 343 \\
\hline
\end{tabular}

Fuente: elaboración propia. 
En el cuadro también se observa la frecuencia de las unidades de significado que se encontraron en los discursos de los estudiantes entrevistados. Para fines de este trabajo, se tomaron en cuenta las cinco (5) categorías de mayor frecuencia, las cuales se sombrearon de color amarillo. Para realizar el análisis de la frecuencia de las unidades de significado y ver la consistencia interna de las categorías, se utilizó el software Atlas Ti.8.

De acuerdo con Vela (2015), el análisis de la información atendió dos aspectos de relevancia, el enfoque inductivo, basado en el procedimiento de Glaser y Strauss, y el uso del Software Atlas Ti.8. Un programa de computación que permite la recolección, reducción y manejo de datos para realizar relaciones y clasificaciones complejas. Además, la construcción y el análisis de las categorías se realizó atendiendo los supuestos básicos del interaccionismo simbólico, abordados en el marco teórico.

En el análisis de resultados, observarán que se entretejieron las unidades de significado del discurso dado por los estudiantes con la interpretación de las investigadoras, puesto que,

las diferentes maneras en que los individuos revisten de significados los objetos, los acontecimientos, las experiencias, etc., forman el punto de partida central de la investigación. La reconstrucción de estos puntos de vista subjetivos se convierte en el instrumento para analizar los mundos sociales (Flick, 2012, p. 32).

La interpretación es la etapa más compleja, sin embargo, "es la aportación original a la investigación, es el resultado de vivir la experiencia de indagación y es la sección que hace valiosa y da significado a la tarea realizada" (Díaz, 2017, p. 37).

\section{Resultados}

\section{Práctica de la investigación}

Esta es una de las categorías que se presenta con mayor consistencia. Los estudiantes reconocen que un trabajo de investigación, no solo debe de ser para acreditar un curso o cumplir un requisito académico, sino una necesidad para tener la práctica, comprender y dar sentido a la teoría; una práctica fundamental para la formación en Pedagogía y en investigación. Al respecto los entrevistados comentaron:

E1FT: algunas prácticas, bueno a mi me favorecieron para saber cómo utilizar cada uno de los métodos en investigación, ya sea cualitativa o cuantitativa.

E2MT: en la metodología de la investigación cualitativa, realicé una encuesta de la escala del estrés laboral que padecen los docentes, y pues ahi aprendí mucho.

E3MT: yo invitaría a la comunidad, bueno más que nada a todos los que estamos estudiando Pedagogía y si desconocen la investigación que erróneamente se apoyen en esto, porque es parte de su formación, que en un momento dado van a tener que hacer una investigación.

Los estudiantes entrevistados también comentaron que, en ocasiones, estas actividades que ayudan a reforzar la práctica de la investigación, suelen ser vistas como algo obligatorio; solo tienen el sentido de acreditar el curso de investigación. Esto se debe al aburrimiento, falta de tiempo e iniciativa para la formación:

E4FT: porque es algo que tenemos que hacer, no tanto por gusto, sino por obligación. 
E7MP: lo que he podido notar es que hacemos investigación por cuestión de una calificación, no tanto por gusto o por placer, o porque nos nazca, sino que lo vemos más como un requerimiento para una calificación o terminar nuestro plan de estudios.

\section{Dificultad para hacer investigación}

Esta categoría tiene una estrecha relación con la categoría anterior. Durante el proceso de formación y conforme se hace la práctica y construcción del trabajo de investigación, al estudiante se le presentan una serie de obstáculos que complican dichos procesos, envolviéndolo en una situación difícil de resolver o superar en su momento, requiriendo a su vez, de un esfuerzo adicional para lograr el objetivo.

E1FT: no quieres hacerlo y aparte la actitud, porque si llegas con una mala actitud de: jay! es que, ¿qué tal si esto, o esto, o esto?, etc. entonces no vas a... va a haber un sesgo de información por parte tuya.

E2MT: pero como tal, como estudiantes, pues no se atreven a poder hacer una ponencia, una estancia académica o sea no lo veo tan fomentada esa parte.

Los estudiantes aluden su dificultad para hacer investigación a los profesores que no han sabido fomentarles el gusto, el interés y la actitud para ello.

E2MT: tenemos maestros que verdaderamente no tienen una buena actitud, o sea no te enseñan como debería de ser, y hay unos que sí.

E3MT: no me he involucrado la verdad, no he tenido asi maestros que me digan o que soliciten un apoyo de mi para que les ayude a investigar, porque no he tenido esa oportunidad... un maestro que está impartiendo el área de investigación, pero no se acerca a su alumno, porque el alumno siempre va a tener dudas, siempre va a tener esas preguntas que quiere que se las respondan, pero por miedo a que un maestro sea asi, de que no tenga actitud y no tenga paciencia con el alumno, entonces el alumno no va a poder perder ese miedo a preguntarle.

E5FP: hay algunos cursos donde con algunos maestros o depende de quién te toque, que se le dedica lo suficiente o la importancia necesaria, porque algunos alumnos como ven que el maestro no le echa muchas ganas, por parte de ellos menos. Sin embargo, a mi me tocaron maestros que no se dedicaron, no tenian esas caracteristicas, no les importaba que sus alumnos aprendieran a investigar.

E6FT: a veces el profesor tal vez no está tan familiarizado con esta parte de la investigación, y tú le preguntas algo y en lugar de facilitarte la respuesta, te enreda más.

Por otro lado, también los estudiantes reconocen su debilidad en aquellos aspectos que son necesarios para formarse en investigación.

E2MT: yo creo que, porque no tenemos como mexicanos esa cultura de estar leyendo, de estar argumentando, escribiendo eldocumento. ... Es muy detallado, tienes que dedicarle tiempo como mencioné hace rato. Entonces pues varios no están acostumbrados a darle tiempo, a darle como que esa importancia de realizar el trabajo de investigación.

E5FP: terminas plagiando, terminas agarrando cosas que no son tuyas en un cien por ciento.

E6FT: muchas de las veces no puedes hacerlo como tal, el salir a investigar o salir a aplicar un instrumento, por cuestiones de economía o dificultades personales.

E7MP: muchas veces suele pasar, que se nos pasa o estamos mal acostumbrados a redactar. No tenemos 
la costumbre de hacer una buena redacción, también lo que mencionabas, no siempre contamos con las posibilidades o la materia prima para llevar a cabo esta investigación; no siempre nos dan nuestros tiempos, no contamos como ya lo decía con el dinero, el material y esto nos puede llegar a poner trabas para realizar una investigación como tal.

E8FP: más que nada son la variedad de palabras que existen, a veces no comprendo una palabra o tal vez una palabra tiene varios significados. Entonces debes de saber cuál es la correcta para entender lo que es el texto completo, más que nada es eso, la palabra si no entiendo, no voy a comprender... al igual si los alumnos tienen flojera o tal vez no les guste o no les interese.

Sin embargo, se puede observar que las dificultades para la práctica de la investigación llevan una relación implicada, tanto del papel que desempaña el docente como el papel que desempeña el estudiante.

\section{La investigación base en la formación}

Por otra parte, a pesar de las dificultades para hacer investigación, los estudiantes identifican claramente al área de investigación, pues conocen los cursos que curricularmente la integran, así también, saben de su relevancia en la formación del profesional de la Pedagogía y sus ventajas en el contexto de la educación.

E1FT: muchos de nosotros no conocemos la manera de investigar y para seguir nuestros estudios, seguir la carrera o especializándonos es necesario saber investigar, saber indagar, saber y conocer métodos y estrategias para tal acción.

E2MT: en el Área está Epistemología y paradigma, Estadistica descriptiva, Estadistica inferencial, Metodología de la investigación cualitativa y cuantitativa, Proyecto de investigación y de ahi viene lo que es la Experiencia recepcional.
E4FT: porque día con dia investigamos ya sea en redes sociales, en nuestra casa, con familiares. Pero en esta Área de investigación, investigamos realmente la educación.

E5FP: porque es una carrera de Humanidades y al final de cuentas, pues es parte y debe ser parte de nosotros como futuros pedagogos, y más si todavía nos titularemos por tesis.

E6FT: porque en esta parte de Humanidades o en cualquier carrera es necesaria hacer la parte de investigación, porque tú necesitas como profesionista que va a indagar, conocer; prácticamente investigar sobre diferentes cosas.

E7MP: considero que las más importantes serian Proyecto de investigación y Experiencia recepcional, porque ya es donde formalizamos más esta cuestión de la investigación... Considero que sí, que es importante que se vea el pedagogo inmerso en la investigación ya que día con día surge el interés y la necesidad de constante preparación, y cómo nos vamos a preparar si no investigamos.

Sin lugar a duda, existe un espacio curricular con identidad para la formación en investigación, entonces cabe preguntarse, ¿por qué el estudiante no tiene el interés por formarse en esta área por iniciativa propia?, ¿por qué ese desinterés?, ¿es acaso el estudiante o es la forma cómo los docentes desarrollan esta área? En la anterior categoría se pudo observar el abordaje de esta situación, sin embargo, existen otros aspectos de contexto social y económico que están presentes, para que el estudiante no tenga el interés de formarse en el área de investigación educativa.

Significado de la investigación

En esta categoría se observa que, para los estudiantes, el significado sobre la investigación se refiere al abordaje de un problema y dar soluciones relacionadas al campo educativo. 
E1FT: para mi la investigación en educación es una respuesta a alguna problemática que se está viviendo día con día.

Si me gusta. Pienso que mientras más conocemos, más podemos darles solución y que la educación es la puerta que puede abrir ese camino a solucionar, como la inseguridad y demás problemas que estamos viviendo.

E3MT: la investigación significa para mí, más que nada, investigar una problemática o algún defecto, si tenemos una problemática educativa entonces investigarla para saber si hay solución o no, o cuáles serian los métodos o estrategias de enseñanza para que esa problemática se pueda resolver.

E5FP: se enfoca en los procesos de enseñanza-aprendizaje y problemáticas reales que existen dentro de la educación.

E6FT: hasta donde yo tengo un conocimiento, la investigación tiene que ver mucho en el campo educativo, porque conoces los problemas sociales. Conoces o investigas e indagas sobre lo que son los problemas en educación, los problemas en las escuelas, de los niños y cosas así.

Otros estudiantes tienen un significado más amplio sobre el término, a saber:

E4FT: pues de esa manera investigando o haciendo por ejemplo la tesis o proyecto de investigación, podemos aportar algo a la educación de nuestras futuras generaciones. Un cambio en nuestra sociedad mediante la investigación, saber cuáles son las fortalezas y debilidades de las nuevas generaciones que hay.

E6FT: cuando entré yo creía que me iba a ir por promedio, entré con esa idea, pero ya estando en la carrera dije: quiero hacer la investigación, porque siento que más que un comodín, es como que esta parte de que es un trabajo, cuando sales allá afuera ya tienes esta parte desarrollada de la investigación.

E7MP: yo considero que si, es muy importante como ya lo habia mencionado, es una pauta o una base que le permite al pedagogo explorar nuevas áreas y poder verse inmerso, abarcar más áreas de trabajo y tenga un amplio campo laboral... es necesario o fundamental que se siga investigando o se siga metiéndose en este tipo de áreas porque ¿de dónde vamos a obtener el conocimiento?, que es todo aquello que vamos a impartir nosotros.

Estos significados sobre investigación se relacionan con la forma en que se aporta algo a la sociedad, como una forma de generar conocimiento; como una parte importante de la formación de un pedagogo que les permitirá tener un mejor campo de trabajo. Sin embargo, otros estudiantes expresaron su puntual desagrado hacia la investigación.

E5FP: primero que nada, de responsabilidad, porque ahora si que hago la investigación, porque es parte de lo que me están pidiendo y dar lo mejor, ser responsable porque en lo personal no me gusta, sin embargo trato de cumplir, ... y la verdad aunque hice el trabajo me vi como parte de esa problemática, porque es cansada, es tediosa y hay que dedicarle demasiado tiempo.

E8FP: siendo sincera me gusta poco, pero es necesario realizarla. Entonces si la llego a hacer, en algunos temas que me llamen la atención, entonces si me pongo a leer y voy haciéndolo, pero asi de manera general, pues la verdad como te digo no del todo.

El significado que el estudiante ha construido sobre la investigación educativa es variado, lo relacionan con un problema y dar soluciones; en aportar conocimiento social y con ideas de desagrado. Estos significados están determinados por las experiencias que el estudiante ha vivido durante su trayectoria escolar. Significados que encuentran relación con el contenido de las categorías anteriores. 
La tesis

En esta categoría se reflejan las experiencias vividas por cada uno de los estudiantes entrevistados y que dan pauta para elegir hacer o no el trabajo recepcional, la tesis, para su titulación. Se encontraron diversos elementos interesantes en el discurso de los estudiantes, cuando se les preguntó sobre el conocimiento que tenían sobre esta.

En cuanto al contenido de la tesis:

E1FT: es un trabajo de investigación en el cual desarrollas una problemática que tú crees existe, en la cual haces una hipótesis de que puede que se rechace o puede que no, porque puede ser cierto o puede ser que no, pero es lo que yo creo... un planteamiento fundamentado, tiene que tener citas me parece que más de cien citas, tiene que tener alrededor de cien páginas, tiene que tener un sustento válido en diversos trabajos anteriores.

E2MT: primero que nada, debe de tener una buena argumentación, un buen sustento teórico, debe de tener una entrevista, un instrumento donde tú vas a investigar y hacer una comparación para ver si, si o no, se cumplen los objetivos o los propósitos.

La tesis es un ejercicio para la práctica de la investigación donde se pone a prueba lo que se aprendió.

E2MT: porque siento que ahi pones en práctica todos tus conocimientos y eso te va a servir cuando te insertes al campo laboral. Es una investigación donde tú vas a indagar sobre un tema, vas a argumentar, vas a defenderlo de alguna u otra forma. Eso sería para mi una tesis.

E3MT: demuestras tu habilidad de redactar, de investigar y de poder analizar, comprender y más que nada aplicar algo serio, algo que realmente le va a servir, puede ser que te sirva a ti, puede ser que a la institución, al que le vas a aplicar o a los que les vas a explicar, entonces, le pueda servir de manera muy práctica, muy significativa para esa persona o esas personas.
E4FT: la tesis es la evaluación de tu carrera, no nada más de tu universidad sino desde el preescolar. Ahi es donde tú mismo te vas a evaluar como persona, cómo puedes ser como persona y como profesionista.

Así también, identifican cuáles son los propósitos de una tesis:

E2MT: tiene un significado, porque obviamente es un trabajo propio o en pareja que obviamente va a impactar y va a dejar algo para la comunidad universitaria, porque se le puede dar seguimiento a esa tesis y de estar satisfecho porque tú hiciste esa investigación, entonces siento que voy a dejar algo muy favorable, productivo para la sociedad.

E4FT: es muy importante, porque te lleva a conocer más, aunque la escuela si te enseña y sales a algunas prácticas, pero como tal no te adentras tanto a lo que es la Experiencia recepcional, sino a lo que es la tesis, al trabajo de campo, a conocer los problemas sociales, los problemas educacionales. Ya en la tesis vas a investigar a fondo, a llevar un tema y salir a ello. Te ayuda a conocer, a experimentar.

E6FT: he escuchado comentarios que realmente me han molestado, porque dicen que tesis para qué, como tú lo habias mencionado, para que perder mi tiempo, y luego hacen tesis que son muchas chafas, que ya están muy usadas y que ya lo vieron. Conforme fui experimentando, pasando la experiencia educativa me llamó la atención hacer este trabajo de campo y preferi hacerlo por esta parte de conocer y más que nada la curiosidad.

Como se puede observar en esta categoría, los estudiantes saben lo que implica realizar una tesis, conocen su estructura y el propósito de esta, sin embargo, relacionando el contexto escolar donde interactúan los estudiantes, la tesis es considerada, por la mayoría de estos, como un trabajo con gran exigencia que requiere de habilidades, conocimiento, gusto y tiempo. Por otro lado, la tesis suele ser un término que está envuelto por cuestiones institucio- 
nales, que la llevan a alejarse de su verdadera naturaleza de formación.

Son pocos los estudiantes que se atreven a desarrollar una tesis, a veces por gusto, a veces porque el promedio nos le ayuda, pero se dan cuenta que es un trabajo que fortalece su formación; quienes la llegan a desarrollar se sienten orgullosos cuando le dan lectura formal.

\section{Conclusiones}

La respuesta a la pregunta de investigación: ¿qué significados ha construido el estudiante de pedagogía en torno a la tesis y la investigación educativa? lleva a considerar que, tales significados se encuentran entrelazados con las categorías que emergieron durante el proceso de interpretación del discurso obtenido por los estudiantes. Desde dichas categorías se presentan las siguientes conclusiones:

Respecto a la práctica de la investigación, los estudiantes reconocen que este aspecto juega un papel fundamental para el desarrollo de habilidades $y$ actitudes indagativas, sin embargo, observan una ausencia de prácticas para hacer investigación, a pesar de contar con un área de investigación educativa en su plan de estudios. Una paradoja en la formación pedagógica.

La práctica de investigación lleva a tomar en cuenta, que los investigadores encuentran la función de la teoría en la práctica, es decir la construcción del habitus tal como acertadamente lo menciona Bourdieu (2003). El habitus lleva a comprender la lógica del campo científico y sobre todo el ejercicio de la investigación, la cual implica práctica;

Una práctica que exige oficio, intuición y sentido práctico, olfato, cosas todas ellas difíciles de transcribir sobre el papel y que sólo pueden ser entendidas y adquiridas realmente mediante el ejemplo y a través de un contacto personal con unas personas competentes (Bourdieu, 2003, p.74).

En este sentido, Brockbank y McGill (2008) consideran que un profesor refuerza la posibilidad de que el alumno participe en la práctica reflexiva cuando modela intencionadamente su procedimiento, que le permita la comprensión de las reglas y prácticas del campo científico.

Contar con espacios para la práctica de investigación, depende, en gran medida, del perfil del profesor, quien debe tener la experiencia en la práctica; no debe ser un profesor estrechamente formal y conceptual, sino al contrario, como lo plantea Sánchez (2014), debe hacer partícipe al investigador en actividades que favorezcan a vivir la experiencia de la investigación, enseñar a hacer investigación desde los propios espacios institucionales donde se promueva la generación del conocimiento científico.

Hacer investigación alude a la forma cómo se enseña. Al respecto, Sánchez (2014) nos menciona cómo trabajar la investigación por la "vía artesanal", porque enseñar a hacer investigación en ciencias sociales y humanidades requiere una comunicación constante y directa entre investigador-estudiante:

A investigar se aprende a lado de otro más experimentado; a investigar se enseña mostrando cómo; a investigar se aprende haciendo, es decir, imitando y repitiendo una y otra vez cada una de las complejas y delicadas labores de la generación de conocimiento: a investigar se enseña corrigiendo; se aprende viendo (Sánchez, 2014, p. 14).

En este sentido, Caramés (2013) también aborda la metáfora del trabajo artesano para generar una significativa experiencia de investigación. Ella argumenta:

comprometerse con un trabajo artesano conlleva el deseo de hacer las cosas bien y estar dispuestos a equivocarnos para aprender... un aprendizaje que se vincula a las experiencias; experiencias que conllevan un disponer a aprender, a arriesgarnos, a equivocarnos y dejarnos llevar por las preguntas que tiran de nosotros (p. 208).

Ante estas interesantes aportaciones, el principio pedagógico "a investigar se aprende investigando" 
cobra un papel fundamental, pues tiene implicaciones importantes en la práctica, en la formación, en la construcción del saber pedagógico.

Por otro lado, los estudiantes reconocieron las dificultades para la práctica de la investigación relacionadas, tanto con el papel del profesor como del propio estudiante.

Respecto a los profesores, los estudiantes coincidieron que hace falta una buena orientación de su parte; un modelaje claro sobre la práctica de la investigación, acompañada de una buena actitud. Este aspecto va relacionado con lo que hemos abordado anteriormente, el perfil del profesor, el cual debe contar con experiencia en la práctica para generar espacios donde se viva la experiencia del aprender a hacer investigación.

Respecto a los estudiantes, ellos mismos están conscientes de las debilidades que tienen para hacer investigación; por ejemplo, respecto a la lectura, escritura y comprensión de palabras que les permita enfrentarse a la construcción propia de su escrito, de su marco teórico; identificar con claridad una temática de investigación; identificar y argumentar su objetivo de estudio.

Esta situación es congruente con el estudio que ha realizado la OCDE, OIE-UNESCO y UNICEF LACRO (2016), para promover los enfoques de investigación para el aprendizaje. Es una tarea compleja pues se observa que la debilidad de los estudiantes con respecto a la comprensión disciplinaria, las capacidades académicas y la gestión de tareas, genera grandes desafíos para el docente y la institución, dificultando la implementación de programas y estrategias para la práctica de la investigación.

Sin embargo, existe otra situación en los estudiantes quienes viven en el mundo de la pseudoconcreción (Kosik, 1967), un claroscuro de verdad y engaño. Pues conociendo el contexto escolar de los estudiantes, estos se dejan atrapar por el conformismo, evadiendo aquellas actividades que sean de mayor exigencia y dedicación.

Otro aspecto que está presente en la dificultad para hacer investigación es el desconocimiento por parte del estudiante sobre aquellos programas que apoyan la formación de jóvenes investigadores, por mencionar algunos programas: Verano de la Investigación Científica de la Academia Mexicana de Ciencias (AMC), Verano de la Investigación Científica y Tecnológica del Pacífico llamado Delfín y, Verano con un Científico, que promueve la propia Universidad Veracruzana. Al interior de la Facultad de Pedagogía existe el Programa para el Fortalecimiento de la Excelencia Educativa (PROFEXCE) de la Secretaría de Educación Pública (SEP), que apoya económicamente la formación integral del estudiante.

Es pertinente agregar a este análisis, aquellos aspectos sociales que envuelven a los estudiantes de la Facultad de Pedagogía de la Universidad Veracruzana. El estado de Veracruz, México, concretamente la Ciudad de Poza Rica y los municipios a su alrededor (Papantla, Coatzintla, Tihuatlán, Cazones, Gutiérrez Zamora, entre otros), son lugares donde aún existen comunidades indígenas (totonacas y nahuatls, principalmente) y con bajos recursos económicos. Esta situación lleva a que los estudiantes tengan como propósito principal al término de su carrera, concursar para una plaza docente. Es lo más inmediato que conocen para su ejercicio profesional y el ingreso económico.

Sin lugar a duda, esta situación social y económica está implicada en la toma de decisiones por parte de los estudiantes, pues consideran que encontrar una plaza como investigadores educativos es una situación lejana; además, conciben la investigación como un área de mayor exigencia profesional que requiere, como ya lo hemos dicho, tiempo y dinero. Aunado a todo esto, la cultura de la tradición en los estudiantes pesa mucho, replicando roles y aspiraciones de sus contextos inmediatos. Al respecto Kincheloe (2001) hace una fuerte crítica:

Los estudiantes de educación entran a menudo en la facultad imbuidos de una serie de expectativas y presuposiciones conservadoras. Aspiran a un devenir de maestros como los que conocieron, para enseñar a alumnos idénticos a como fueran ellos mismos y sus compañeros... De este modo los alumnos de educación no tienden 
a ser buscadores de formas alternativas de ver ni de ópticas que les permitan reconceptualizar el conocimiento y la pedagogía (p. 27).

México es un país centralista en el campo de la investigación educativa, pese a los esfuerzos realizados para superar esta condición, tanto por el Consejo Mexicano de Investigación Educativa (COMIE) como por las propias universidades públicas y privadas en los diferentes estados del país, la tarea es difícil y compleja (De Alba, 2010).

La cobertura del financiamiento para hacer la investigación y la formación de jóvenes investigadores educativos en las diversas instituciones de educación superior en el país sigue siendo aún muy débil. Aunado a ello, la fragmentación docencia-investigación aún presente; por un lado, existen los institutos o centro dedicados a la investigación, y por el otro, las Facultades o Escuelas donde se hace docencia, creciendo así la tensión y la contradicción entre el diseño de las políticas y la vida cotidiana de las escuelas (Sverdlick, 2012).

Por otra parte, a pesar de esta situación, los estudiantes reconocen la investigación como base en la formación; conocen los cursos que curricularmente la integran, así como su relevancia de la formación del profesional de la Pedagogía, y su aplicación en el contexto de la educación.

Sin lugar a duda, existe un espacio curricular con identidad para la formación en investigación, entonces cabe preguntarse, ¿cómo hacer para que el estudiante despierte su interés por formarse en esta área?, ¿qué estrategias diseñar para fortalecer la formación y el currículum escolar? Como lo abordamos anteriormente, existen cuestiones sociales y económicas que interfieren para que el estudiante se interese por la investigación educativa, sin embargo, es de reconocerse que hace falta un sólido trabajo colegiado con miras a fortalecer la formación.

Se requiere innovar el espacio curricular y académico no solo en el área de la investigación educativa, sino de todo el plan de estudios de la carrera de Pedagogía, con estrategias transversales en el trabajo académico orientadas a gestionar espacios que favo- rezcan las experiencias de aprendizajes y el desarrollo de las habilidades investigativas.

$\mathrm{Al}$ respecto, existen recientes experiencias en algunas universidades mexicanas que están implementado el Aprendizaje Basado en Investigación (ABI), como estrategia para fortalecer la formación en investigación, tanto en programas de licenciatura como en el posgrado. El ABI es una propuesta que hizo Ernest Boyer para que un estudiante universitario participara en actividades de investigación (Figueroa, Reyes y Fiorentini, 2018; Torres, s.f.).

Una de las principales universidades privadas en México, pionera en el contexto latinoamericano sobre el trabajo del ABI, es el Tecnológico de Monterrey, siendo impulsor de esta estrategia el Dr. Arturo Torres Tobías quien concibe al ABI como:

un enfoque didáctico que permite hacer uso de estrategias de aprendizaje activo para desarrollar en el estudiante competencias que le permitan realizar una investigación creativa en el mundo del conocimiento (Torres, s.f., p. 2)

El Dr. Torres diseñó un programa de habilidades docentes para trabajar con el ABI, que permita vincular la investigación con los programas académicos y las actividades de enseñanza.

Por otro lado, se encuentra la experiencia de la Universidad Quintana Roo, universidad pública que ha implementado el ABI para fortalecer la relación docencia-investigación en los programas de licenciatura en educación superior (Véase, Figueroa et al., 2018).

Existen otras experiencias en universidades del Ecuador, donde esta estrategia ha sido de interés para fortalecer los programas del posgrado y la licenciatura (Véase, Peñaherrera, Chiluiza y Ortiz, 2014; Gordillo, Gordillo y Campuzano, 2017).

Estos ejemplos permiten ver que una indagación sistemática y autocrítica requiere de estrategias, pues "la indagación, se halla basada en la curiosidad y en un deseo de comprender; pero se trata de una curiosidad estable, no fugaz. Sistemática en el sentido de 
hallarse respaldada por una estrategia" (Stenhouse, 2004 , p. 28), permitiendo llegar a la llamada curiosidad epistemológica (Freire, 2018).

En este sentido, se abre todo un desafío para los profesores, los estudiantes, la institución y el propio currículum, pues "los enfoques exitosos basados en la indagación requieren de planificación y enfoques bien pensados sobre colaboración, la interacción en el aula y la evaluación" (OCDE, OIE-UNESCO, UNICEF LACRO, 2016, p. 173).

Por último, encontramos una categoría que su contenido se entrelaza con las anteriores, el significado de la investigación. El significado que el estudiante ha construido sobre la investigación educativa es diverso, tiene que ver con un problema, soluciones, en aportar conocimiento social y con ideas de desagrado. Estos significados están determinados por las experiencias que el estudiante ha vivido durante su trayectoria escolar y la interacción que ha establecido con los miembros de su comunidad universitaria.

Sin embargo, en tales significados también encontramos la enunciación de una práctica en ocasiones ausente, relacionada con la forma como el docente modela la forma de hacer investigación y la apertura para ello. Estos aspectos hacen que el estudiante considere a la investigación como difícil, pesada y aburrida.

Por otro lado, encontramos otros aspectos que están determinados por la cuestión institucional, un significado que connota tiempo y "un tener que" cumplir con los cursos que marca la malla curricular.

Ante tal situación, es de reconocerse que la formación en investigación consiste en incluir una participación más consciente de quien se forma en la cultura científico-disciplinar, ya que a través de las distintas experiencias en las cuales se ven involucrados los estudiantes durante el proceso, hacen que asimilen y se comprometan de una forma más responsable con su propia formación. Una formación que, como pedagogos, lleva a tener presente que la enseñanza exige investigación (Freire, 2018). Aspecto que debe caracterizar el proceso de formación del estudioso de la educación: el pedagogo.
La formación en investigación educativa debe ser una condición necesaria en la formación de los jóvenes universitarios, de acuerdo con CochranSmith y Lytle (2002), la investigación es una actividad importante para la actividad educadora. La investigación es educativa en el grado en que puede relacionarse con la práctica de la educación (Stenhouse, 2004).

Por último, surgió una categoría que determina el alcance y las limitaciones de formación de un estudiante, la tesis. Los estudiantes de la Facultad de Pedagogía saben lo que implica realizar una tesis, conocen su estructura y su propósito, sin embargo, desde el contexto escolar donde interactúan, la tesis es un trabajo considerado por ellos mismos de gran exigencia que requiere de sólidas habilidades para escribir, leer e indagar; de conocimiento amplio, de gusto y tiempo. Además, es un término que está envuelto por cuestiones institucionales que lo llevan a alejarse de su verdadera naturaleza de formación.

Ante el peso institucional y las connotaciones negativas que los estudiantes suelen atribuirle a la tesis, cabe hacer la reflexión sobre el papel que ésta juega, en el proceso de formación de un estudiante. La tesis es el reflejo del acto de estudiar, en palabras de Freire es "realmente, un trabajo difícil. Exige de quien lo hace una postura crítica, sistemática. Exige una disciplina intelectual que no se adquiere sino practicándola” (Frerie, 2011, p. 48). Una práctica reflexiva que promueva el aprendizaje, la investigación, el estudio, la experiencia afectiva y cognitiva (Brockbank y McGill, 2008).

La tesis exige un trabajo intelectual, es el resultado de un proceso de escritura y re-escritura; es un ir y venir de pensamientos y reflexiones que llevan a encontrar un posicionamiento teórico y crítico. La tesis es la oportunidad, el espacio y el tiempo para encontrar el verdadero sentido y significado de la experiencia durante el acto de estudiar.

En el contexto de la Facultad de Pedagogía, son pocos los estudiantes que se atreven a desarrollar una tesis, a veces por gusto, a veces porque el promedio nos le ayuda, cualquiera que sea su situación, se dan cuenta que es un trabajo para fortalecer su formación, brin- 
dando un orgullo y satisfacción cuando la presentan ante los académicos, la institución y su familia.

A partir de las conclusiones realizadas, se proponen algunas recomendaciones para mejorar la formación en investigación educativa, no solo en los estudiantes de la Facultad de Pedagogía de la Universidad Veracruzana, México, sino también de otros espacios académicos.

Cuidar el perfil del profesorado es fundamental para el área de investigación educativa, dado que, la elección del estudiante, tanto para hacer una tesis como para incursionar en el área de la investigación educativa, dependerá de cómo el profesor despierte en él su inquietud, su interés y curiosidad epistémica.

Diseñar estrategias transversales por parte del profesorado, para fomentar el desarrollo de habilidades y actitudes indagativas en los estudiantes. Como ejemplo ya abordado, la implementación del Aprendizaje Basado en Investigación.

Considerar la tesis como parte de la estrategia para la formación en investigación; como un espacio para realizar diversas prácticas que involucren su desarrollo gradual, que genere la experiencia de un aprendizaje significativo para su formación y desarrollo profesional.

Generar proyectos de investigación desde los diversos grupos de profesores que permitan, ser oportunidades de formación tanto para los mismos académicos como para los estudiantes, impulsando el desarrollo curricular del área de la investigación educativa y de todo el plan de estudios.

Establecer redes de colaboración y movilidad académica, que permitan ser un puente para que el estudiante realice estancias de investigación a nivel nacional e internacional, ampliando su visión en la formación profesional.

El diseño para el cambio en las políticas educativas y de investigación debe hacerse desde los actores principales del currículum: profesores y estudiantes, tomando en cuenta una mirada in situ de las características de los diversos contextos académicos, si en realidad se quiere fortalecer el quehacer científico, la investigación educativa y la educación superior en México.

\section{Referencias}

Aguirre, J. (2020). La posibilidad de la objetividad en ciencias humanas. Recuperado de: https:// scielo.conicyt.cl/pdf/cmoebio/n67/0717554X-cmoebio-67-00001.pdf

Blumer, H. (1982). El interaccionismo simbólico: perspectiva y método. Barcelona: Hora, S.A.

Bourdieu, P. (2003). El oficio del científico. Ciencia de la ciencia y reflexividad. España: Anagrama.

Brockbank, A. y McGill, I. (2008). Aprendizaje reflexivo en la educación superior. Madrid: Morata.

Caramés, B. (2013). Investigar en educación: un espacio posible y sobre todo imposible. En J. Contreras y D. L. Pérez. (Comps). Investigar la experiencia educativa (pp. 199-210). Madrid: Morata.

Cochran-Smith, M. y Lytle, S. (2002). Dentro/Fuera. Enseñantes que Investigan. Madrid: Akal.

Contreras, J. y Pérez, D. L. (2013). Investigar la experiencia educativa. Madrid: Morata.

De Alba, A. (2010). Investigación educativa ¿¿De la superficie sedimentada y firme a la pérdida de piso? Reflexiones a partir del año 2010 en México. En Nowalski (Coord.). En el camino de la titulación. Trazos, tesis y tramos (pp.155180). México: UNAM/Díaz de Santos.

Denzin, N. y Lincoln, Y. (2011). Manual de Investigación Cualitativa. Volumen I. México: Gedisa.

Díaz, B. (2017). La interpretación en la investigación educativa. En B. Díaz, y C. Domínguez. (Coords.). Interpretación: un reto en la investigación educativa (pp.15-38). México: AUTxNewton.

Eisner, E. (1998). El ojo ilustrado. Indagación cualitativa y mejora de la práctica educativa. España: Paidós.

EPISTEMOLOGÍA, CONSENTIMIENTO INFORMADO, ENTREVISTAS EN PRODUN$D I D A D$. Ecuador: Ibarra-Ecuador. Recuperado de https://www.studocu.com/es/document/ universidad-camilo-jose-cela/marketing/trabajo-tutorial/libro-de-investigacion-cualitativa-digital-compressed/4751385/view 
Facultad de Pedagogía. (2001). Plan de Estudios Pedagogía 2000. Universidad Veracruzana. Recuperado de: https://www.uv.mx/sea/ files/2012/10/pedagogia.pdf

Figueroa, M., Reyes, D. y Fiorentini, N. (enero-julio, 2018). El aprendizaje basado en la investigación (ABI) como un factor para el fortalecimiento de los programas educativos de la Universidad Quintana Roo en Playa del Carmen, México. Ensayos Pedagógicos, XIII(1),131-156. Recuperado de file://C:/Users/HP\%20\%20ENVY\%20 84856/Downloads/El_aprendizaje_basado_en_ la_investigacion_ABI_como.pdf

Flick, U. (2012). Introducción a la investigación educativa. Madrid: Morata.

Freire, P. (2018). Pedagogía de la autonomía. Saberes necesarios para la práctica educativa. México: Siglo XXI Editores.

Freire, P. (2011). La importancia de leer y el proceso de liberación. México: Siglo XXI Editores.

Glaser, B. y Strauss, A. (1967). The Discovery of Grounded Theory. Strategies for Qualitative Research. Chicago: Aldine Transaction.

Gordillo, M., Gordillo, M. y Campuzano, V. (noviembre, 2017). La docencia con enfoque de investigación para el aprendizaje. YACHANA, 6(2), 65-75. Recuperado de http://repositorio. ulvr.edu.ec/bitstream/44000/3588/1/La\%20 docencia $\% 20$ con $\% 20$ enfoque $\% 20$ de $\% 20$ investigaci \%c3\%b3n\%20para\%20el\%20 aprendizaje.pdf

Kosik, K. (1967). Dialéctica de lo concreto. Recuperado de http://theomai.unq.edu.ar/conflictos_sociales/ Kosik_Dial\%C3\%A9ctica_de-lo_Concreto_ cap1.pdf

Kincheloe, J. (2001). Hacia una revisión critica del pensamiento docente. España: Octaedro.

Kvale, S. (2011). Las entrevistas en Investigación Cualitativa. Madrid: Morata.

Martínez, M. (2007). La investigación cualitativa etnográfica en Educación. Manual teórico-práctico. México: Trillas.
Organización para la Cooperación y el Desarrollo Económicos (OCDE),

Oficina Regional para América Latina y el Caribe de UNICEF (UNICEF-LACRO) y la Oficina Internacional de Educación de la UNESCO (OIE-UNESCO). (2016). La naturaleza del aprendizaje: usando la investigación para inspirar la práctica. Recuperado de http://panorama. oei.org.ar/_dev/wpcontent/uploads/2017/09/ UNICEF_UNESCO_OECD_Naturaleza_ Aprendizaje_.pdf

Peñaherrera, M., Chiluiza, K., Ortiz, A. (2014). Inclusión del Aprendizaje Basado en Investigación (ABI) como práctica pedagógica en el diseño de postgrados en Ecuador. Elaboración de una propuesta. Journals for Educators, Teachers and Trainers, 5(2), 204-220. Recuperado de http:// www.ugr.es/ jett/pdf/Vol5(2)_015_jett_Penaherrera_Chiluiza_Ortiz.pdf

Programa de Desarrollo de Habilidades Docentes. TEC de Monterrey. Recuperado de http:// www.itesca.edu.mx/documentos/desarrollo_ academico/Metodo_Aprendizaje_Basado_en_ Investigacion.pdf

Sánchez, R. (2014). Enseñar a investigar. Una didáctica nueva de la investigación en ciencias sociales y humanas. México: Universidad Autónoma Metropolitana.

Stenhouse, L. (2003). Investigación y desarrollo del curriculum. Madrid: Morata.

Stenhouse, L. (2004). La Investigación como base de la enseñanza. Madrid: Morata.

Sverdlick, I. (2012). La investigación educativa como instrumento de acción, formación y de cambio. En Sverdlick, I. (Comp.). La investigación educativa. Una herramienta de conocimiento y acción (pp. 15-46). Argentina: Noveduc.

Taylor, S. y Bogdan, R. (2000). Introducción a los métodos cualitativos de investigación. España: Paidós Básica.

Torres, A. (s.f). Aprendizaje Basado en la Investigación. Técnicas didácticas. 
Trujillo, C., Naranjo, M., Lomas, K., y Merlo, M. (2019). Investigación Cualitativa. Universidad Veracruzana. (2008). Legislación Universitaria. Estatutos de Alumnos. Xalapa, Veracruz: Universidad Veracruzana. Recuperado de https://www.uv.mx/legislacion/files/2012/12/ estatutodelosalumnos2008.pdf
Vela, F. (2015). Un acto metodológico básico de la investigación social: la entrevista cualitativa. En M. Tarrés. (Coord.). OBSERVAR ESCUCHAR Y COMPRENDER sobre la tradición cualitativa en la investigación social (pp. 63-92). México: El Colegio de México/FLACSO. 